\title{
Uniform Asymptotic Stability of Solutions of Fractional Functional Differential Equations
}

\author{
Yajing Li and Yejuan Wang \\ School of Mathematics and Statistics, Lanzhou University, Lanzhou 730000, China \\ Correspondence should be addressed to Yejuan Wang; wangyj@lzu.edu.cn
}

Received 30 September 2013; Accepted 15 October 2013

Academic Editor: Massimiliano Ferrara

Copyright (C) 2013 Y. Li and Y. Wang. This is an open access article distributed under the Creative Commons Attribution License, which permits unrestricted use, distribution, and reproduction in any medium, provided the original work is properly cited.

Some global existence and uniform asymptotic stability results for fractional functional differential equations are proved. It is worth mentioning that when $\alpha=1$ the initial value problem $D^{\alpha}\left[y(t) e^{\beta t}\right]=f\left(t, y_{t}\right) e^{\beta t}, t \in\left[t_{0}, \infty\right), t_{0} \geqslant 0,0<\alpha<1, y(t)=$ $\phi(t), t_{0}-h \leqslant t \leqslant t_{0}$, reduces to a classical dissipative differential equation with delays as in Caraballo et al.s work (2005).

\section{Introduction}

Consider the initial value problem (IVP for short) of the following fractional functional differential equation:

$$
\begin{gathered}
D^{\alpha}\left[y(t) e^{\beta t}\right]=f\left(t, y_{t}\right) e^{\beta t}, \quad t \in\left[t_{0}, \infty\right), t_{0} \geqslant 0, \\
0<\alpha<1, \\
y(t)=\phi(t), \quad t_{0}-h \leqslant t \leqslant t_{0},
\end{gathered}
$$

where $D^{\alpha}$ is the Caputo fractional derivative, $\beta>0, f: J \times$ $C([-h, 0], \mathbb{R}) \rightarrow \mathbb{R}$, where $J=\left[t_{0}, \infty\right)$, is a given function satisfying some assumptions that will be specified later, $h>0$, and $\phi \in C\left(\left[t_{0}-h, t_{0}\right], \mathbb{R}\right)$. If $y \in C\left(\left[t_{0}-h, \infty\right), \mathbb{R}\right)$, then for any $t \in\left[t_{0}, \infty\right)$, define $y_{t}$ by

$$
y_{t}(\theta)=y(t+\theta), \quad \theta \in[-h, 0] .
$$

The study of retarded differential equations is an important area of applied mathematics due to physical reasons, noninstant transmission phenomena, memory processes, and specially biological motivations (see, e.g., [1-4]). Fractional differential equations have attracted much attention recently (see, e.g., [5-11] and the references cited therein for the applications in various sciences such as physics, mechanics, chemistry, and engineering).

Some attractive results for fractional functional differential equations and nonlinear functional integral equations are obtained by using the fixed point theory; see [1216] and references therein. Global asymptotic stability of solutions of a functional integral equation is discussed in [17]; however, there is no work on uniform asymptotic stability of solutions of fractional functional differential equation. It is our intention here to show the global existence and uniform asymptotic stability of the fractional functional differential equation (1).

We organize the paper as follows. In Section 2, we recall some necessary concepts and results. In Section 3 we give the global existence and uniform asymptotic stability of fractional functional differential equations. Finally, two examples are given to illustrate our main results.

\section{Preliminaries}

In this section, we introduce notations, definitions, and preliminary facts which are used throughout this paper.

We consider $B C:=B C\left(\left[t_{0}-h, \infty\right), \mathbb{R}\right)$ the Banach space of all bounded and continuous functions from $\left[t_{0}-h, \infty\right)$ into $\mathbb{R}$ with the norm

$$
\|y\|_{\infty}:=\sup \left\{|y(t)|: t \in\left[t_{0}-h, \infty\right)\right\}
$$

Let $\left\|y_{t}\right\|=\sup _{-h \leqslant \theta \leqslant 0}|y(t+\theta)|$ for $t \in J$.

Throughout this paper, we always assume that $f\left(t, x_{t}\right)$ satisfies the following condition: 
$\left(H_{0}\right) f\left(t, x_{t}\right)$ is Lebesgue measurable with respect to $t$ on $\left[t_{0}, \infty\right)$, and $f(t, \varphi)$ is continuous with respect to $\varphi$ on $C([-h, 0], \mathbb{R})$.

By condition $\left(H_{0}\right)$ and the technique used in [7], we get the equivalent form of IVP (1) as

$$
\begin{aligned}
& y(t) \\
& =\left\{\begin{array}{cl}
y\left(t_{0}\right) e^{-\beta\left(t-t_{0}\right)} \\
+\frac{1}{\Gamma(\alpha)} \int_{t_{0}}^{t}(t-s)^{\alpha-1} e^{-\beta(t-s)} \\
\times f\left(s, y_{s}\right) d s, \quad t \geqslant t_{0}, \\
\phi(t), & t \in\left[t_{0}-h, t_{0}\right],
\end{array}\right.
\end{aligned}
$$

where $\Gamma(\cdot)$ is the gamma function.

Definition 1. We say that the solutions $y$ and $x$ of IVP (1) are uniformly asymptotically stable if for any bounded subset $B$ of $C([-h, 0], \mathbb{R})$ and $\varepsilon>0$, there exists a $T>0$ such that

$$
\begin{aligned}
\left|y\left(t, t_{0}, \phi\right)-x\left(t, t_{0}, \psi\right)\right| \leqslant \varepsilon & \forall t \geqslant T, \\
& \phi, \psi \in B .
\end{aligned}
$$

We recall the following generalization of Gronwall's lemma for singular kernels [18], which will be used in the sequel.

Lemma 2. Let $v:\left[t_{0}, b\right] \rightarrow[0,+\infty)$ be a real function and $w(\cdot)$ is a nonnegative, locally integrable function on $\left[t_{0}, b\right]$ and there are constants $a>0$ and $0<\alpha<1$ such that

$$
v(t) \leqslant w(t)+a \int_{t_{0}}^{t} \frac{v(s)}{(t-s)^{\alpha}} d s .
$$

Then there exists a constant $K=K(\alpha)$ such that

$$
v(t) \leqslant w(t)+K a \int_{t_{0}}^{t} \frac{w(s)}{(t-s)^{\alpha}} d s
$$

for every $t \in\left[t_{0}, b\right]$.

Theorem 3 (Leray-Schauder fixed-point theorem). Let $P$ be a continuous and compact mapping of a Banach space $X$ into itself, such that the set

$$
\{x \in X: x=\lambda P x \text { for some } 0 \leqslant \lambda \leqslant 1\} \text {, }
$$

is bounded. Then, $P$ has a fixed point.

\section{FDEs of Fractional Order}

In this section, we will investigate the IVP (1). Our first global existence and uniform asymptotic stability result for the IVP (1) is based on the Banach contradiction principle and Lemma 2.
Theorem 4. Assume that $f\left(t, y_{t}\right)$ satisfies conditions $\left(H_{0}\right)$ and $\left(H_{1}\right)$ there exists $l>0$ such that

$$
\left|f\left(t, u_{t}\right)-f\left(t, v_{t}\right)\right| \leqslant l\left\|u_{t}-v_{t}\right\|
$$

for $t \in J$ and every $u_{t}, v_{t} \in C([-h, 0], \mathbb{R})$. Moreover, the function $t \mapsto f(t, 0)$ is bounded with $f_{0}=\sup _{t \geqslant t_{0}}|f(t, 0)|$.

If

$$
l\left(\frac{\left(t_{0}+h\right)^{\alpha-1} e^{-\beta\left(t_{0}+h\right)}}{\beta \Gamma(\alpha)}+\frac{\left(t_{0}+h\right)^{\alpha}}{\Gamma(\alpha+1)}\right)<1,
$$

then the IVP (1) has a unique solution in the space BC. Moreover, solutions of IVP (1) are uniformly asymptotically stable.

Proof. We divide the proof into two steps.

Step 1 . We define the operator $P: C\left(\left[t_{0}-h, \infty\right), \mathbb{R}\right) \rightarrow C\left(\left[t_{0}-\right.\right.$ $h, \infty), \mathbb{R})$ by

$$
\begin{aligned}
& (P y)(t) \\
& =\left\{\begin{array}{cl}
y\left(t_{0}\right) e^{-\beta\left(t-t_{0}\right)} \\
+\frac{1}{\Gamma(\alpha)} \int_{t_{0}}^{t}(t-s)^{\alpha-1} e^{-\beta(t-s)} \\
\times f\left(s, y_{s}\right) d s, & t \geqslant t_{0}, \\
\phi(t), & t \in\left[t_{0}-h, t_{0}\right] .
\end{array}\right.
\end{aligned}
$$

The operator $P$ maps $B C$ into itself. Indeed for each $y \in B C$, and for each $t \geqslant 2 t_{0}+h$, it follows from $\left(H_{1}\right)$ that

$$
\begin{aligned}
& |(P y)(t)| \\
& \leqslant\left|y\left(t_{0}\right)\right| e^{-\beta\left(t-t_{0}\right)} \\
& \quad+\frac{1}{\Gamma(\alpha)} \int_{t_{0}}^{t}(t-s)^{\alpha-1} e^{-\beta(t-s)}\left(f_{0}+l\left\|y_{s}\right\|\right) d s \\
& \leqslant\|\phi\| e^{-\beta\left(t-t_{0}\right)}+\frac{f_{0}+l\|y\|_{\infty}}{\Gamma(\alpha)} \\
& \quad \times\left(\int_{t_{0}}^{t-\left(t_{0}+h\right)}\left(t_{0}+h\right)^{\alpha-1} e^{-\beta(t-s)} d s+\int_{t-\left(t_{0}+h\right)}^{t}(t-s)^{\alpha-1} d s\right) \\
& \leqslant\|\phi\|+\left(f_{0}+l\|y\|_{\infty}\right)\left(\frac{\left(t_{0}+h\right)^{\alpha-1} e^{-\beta\left(t_{0}+h\right)}}{\beta \Gamma(\alpha)}+\frac{\left(t_{0}+h\right)^{\alpha}}{\Gamma(\alpha+1)}\right) .
\end{aligned}
$$

For each $t \in\left[t_{0}, 2 t_{0}+h\right]$, we have

$$
|(P y)(t)| \leqslant\|\phi\|+\frac{\left(f_{0}+l\|y\|_{\infty}\right)\left(t_{0}+h\right)^{\alpha}}{\Gamma(\alpha+1)},
$$

and consequently $P(y) \in B C$. 
Since $B C:=B C\left(\left[t_{0}-h, \infty\right), \mathbb{R}\right)$ is a Banach space with norm $\|\cdot\|_{\infty}$, we will show that $P: B C \rightarrow B C$ is a contraction map. Let $y_{1}, y_{2} \in B C$. Then, we have for each $t \geqslant t_{0}$,

$$
\begin{aligned}
& \left|\left(P y_{1}\right)(t)-\left(P y_{2}\right)(t)\right| \\
& \leqslant \frac{1}{\Gamma(\alpha)} \int_{t_{0}}^{t}(t-s)^{\alpha-1} e^{-\beta(t-s)} \\
& \quad \times\left|f\left(s, y_{1 s}\right)-f\left(s, y_{2 s}\right)\right| d s \\
& \leqslant \frac{l}{\Gamma(\alpha)} \int_{t_{0}}^{t}(t-s)^{\alpha-1} e^{-\beta(t-s)}\left\|y_{1 s}-y_{2 s}\right\| d s .
\end{aligned}
$$

Therefore, for any $t \geqslant 2 t_{0}+h$,

$$
\begin{aligned}
& \left|\left(P y_{1}\right)(t)-\left(P y_{2}\right)(t)\right| \\
& \leqslant \frac{l}{\Gamma(\alpha)}\left\|y_{1}(\cdot)-y_{2}(\cdot)\right\|_{\infty} \\
& \quad \times\left(\int_{t_{0}}^{t-\left(t_{0}+h\right)}\left(t_{0}+h\right)^{\alpha-1} e^{-\beta(t-s)} d s\right. \\
& \left.\quad+\int_{t-\left(t_{0}+h\right)}^{t}(t-s)^{\alpha-1} d s\right) \\
& \leqslant l\left(\frac{\left(t_{0}+h\right)^{\alpha-1} e^{-\beta\left(t_{0}+h\right)}}{\beta \Gamma(\alpha)}+\frac{\left(t_{0}+h\right)^{\alpha}}{\Gamma(\alpha+1)}\right) \\
& \quad \times\left\|y_{1}(\cdot)-y_{2}(\cdot)\right\|_{\infty},
\end{aligned}
$$

and for $t_{0}-h \leqslant t \leqslant 2 t_{0}+h$,

$$
\begin{aligned}
& \left|\left(P y_{1}\right)(t)-\left(P y_{2}\right)(t)\right| \\
& \quad \leqslant \frac{l}{\Gamma(\alpha)}\left\|y_{1}(\cdot)-y_{2}(\cdot)\right\|_{\infty} \int_{t_{0}}^{t}(t-s)^{\alpha-1} d s \\
& \quad \leqslant \frac{l\left(t_{0}+h\right)^{\alpha}}{\Gamma(\alpha+1)}\left\|y_{1}(\cdot)-y_{2}(\cdot)\right\|_{\infty},
\end{aligned}
$$

and thus

$$
\begin{aligned}
& \left\|\left(P y_{1}\right)(\cdot)-\left(P y_{2}\right)(\cdot)\right\|_{\infty} \\
& \leqslant l\left(\frac{\left(t_{0}+h\right)^{\alpha-1} e^{-\beta\left(t_{0}+h\right)}}{\beta \Gamma(\alpha)}+\frac{\left(t_{0}+h\right)^{\alpha}}{\Gamma(\alpha+1)}\right) \\
& \quad \times\left\|y_{1}(\cdot)-y_{2}(\cdot)\right\|_{\infty} .
\end{aligned}
$$

Hence, (10) and (17) imply that the operator $P$ is a contraction. Therefore, $P$ has a unique fixed point by Banach's contraction principle.
Step 2. For any two solutions $x=x(t)$ and $y=y(t)$ of IVP (1) corresponding to initial values $\psi$ and $\phi$, by (4) we can deduce that for all $t \geqslant t_{0}+h$ and all $\theta \in[-h, 0]$,

$$
\begin{aligned}
& |x(t+\theta)-y(t+\theta)| \\
& \leqslant\left|x\left(t_{0}\right)-y\left(t_{0}\right)\right| e^{-\beta\left(t+\theta-t_{0}\right)} \\
& \quad+\frac{1}{\Gamma(\alpha)} \int_{t_{0}}^{t+\theta}(t+\theta-s)^{\alpha-1} e^{-\beta(t+\theta-s)} \\
& \quad \times\left|f\left(s, x_{s}\right)-f\left(s, y_{s}\right)\right| d s \\
& \leqslant\left|x\left(t_{0}\right)-y\left(t_{0}\right)\right| e^{-\beta\left(t+\theta-t_{0}\right)} \\
& \quad+\frac{l}{\Gamma(\alpha)} \int_{t_{0}}^{t+\theta}(t+\theta-s)^{\alpha-1} e^{-\beta(t+\theta-s)}\left\|x_{s}-y_{s}\right\| d s .
\end{aligned}
$$

Then, it follows that

$$
\begin{aligned}
e^{\beta t} \| & x_{t}-y_{t} \| \\
\leqslant & \left|x\left(t_{0}\right)-y\left(t_{0}\right)\right| e^{\beta\left(h+t_{0}\right)} \\
& +\frac{l e^{\beta h}}{\Gamma(\alpha)} \int_{t_{0}}^{t}(t-s)^{\alpha-1} e^{\beta s}\left\|x_{s}-y_{s}\right\| d s .
\end{aligned}
$$

Let $w(t)=e^{\beta t}\left\|x_{t}-y_{t}\right\|$. Then, we have

$$
\begin{aligned}
w(t) \leqslant & \left|x\left(t_{0}\right)-y\left(t_{0}\right)\right| e^{\beta\left(h+t_{0}\right)} \\
& +\frac{l e^{\beta h}}{\Gamma(\alpha)} \int_{t_{0}}^{t}(t-s)^{\alpha-1} w(s) d s .
\end{aligned}
$$

Applying Lemma 2, one can see that there exists a constant $K$ such that

$w(t)$

$$
\begin{aligned}
\leqslant & \left|x\left(t_{0}\right)-y\left(t_{0}\right)\right| e^{\beta\left(h+t_{0}\right)} \\
& +\frac{K l e^{\beta h}}{\Gamma(\alpha)} \int_{t_{0}}^{t}(t-s)^{\alpha-1}\left|x\left(t_{0}\right)-y\left(t_{0}\right)\right| e^{\beta\left(h+t_{0}\right)} d s \\
\leqslant & \left|x\left(t_{0}\right)-y\left(t_{0}\right)\right| e^{\beta\left(h+t_{0}\right)}\left(1+\frac{K l e^{\beta h}}{\Gamma(\alpha+1)}\left(t-t_{0}\right)^{\alpha}\right) .
\end{aligned}
$$

Hence, we obtain

$$
\begin{aligned}
e^{\beta t}\left\|x_{t}-y_{t}\right\|= & w(t) \\
\leqslant & \left|x\left(t_{0}\right)-y\left(t_{0}\right)\right| e^{\beta\left(h+t_{0}\right)} \\
& \times\left(1+\frac{K l e^{\beta h}}{\Gamma(\alpha+1)}\left(t-t_{0}\right)^{\alpha}\right),
\end{aligned}
$$

and thus for all $t \geqslant t_{0}+h$,

$$
\begin{aligned}
& |x(t)-y(t)| \\
& \quad \leqslant\left|x\left(t_{0}\right)-y\left(t_{0}\right)\right| e^{-\beta\left(t-h-t_{0}\right)}\left(1+\frac{K l e^{\beta h}}{\Gamma(\alpha+1)}\left(t-t_{0}\right)^{\alpha}\right),
\end{aligned}
$$

which implies that the solutions of IVP (1) are uniformly asymptotically stable. 
Now we give global existence and uniform asymptotic stability results based on the nonlinear alternative of LeraySchauder type.

Theorem 5. Assume that the following hypotheses hold:

$\left(\mathrm{H}_{2}\right) \mathrm{f}$ is a continuous function;

$\left(H_{3}\right)$ there exist positive functions $k_{1}, k_{2} \in B C\left(\left[t_{0}, \infty\right), \mathbb{R}_{+}\right)$ such that

$$
\left|f\left(t, u_{t}\right)\right| \leqslant k_{1}(t)+k_{2}(t)\left\|u_{t}\right\|
$$

for $t \in J$ and every $u_{t} \in C([-h, 0], \mathbb{R})$;

$\left(\mathrm{H}_{4}\right)$ moreover, assume that

$$
\begin{aligned}
& \lim _{t \rightarrow \infty} \int_{t_{0}}^{t}(t-s)^{\alpha-1} e^{-\beta(t-s)} k_{1}(s) d s=0, \\
& \lim _{t \rightarrow \infty} \int_{t_{0}}^{t}(t-s)^{\alpha-1} e^{-\beta(t-s)} k_{2}(s) d s=0 .
\end{aligned}
$$

Then the IVP (1) admits a solution in the space BC. Moreover, solutions of IVP (1) are uniformly asymptotically stable.

Proof. Let $P: C\left(\left[t_{0}-h, \infty\right), \mathbb{R}\right) \rightarrow C\left(\left[t_{0}-h, \infty\right), \mathbb{R}\right)$ be defined as in (11). First, we show that $P$ maps $B C$ into itself. Let $K_{1}=\sup _{t \geqslant t_{0}} k_{1}(t), K_{2}=\sup _{t \geqslant t_{0}} k_{2}(t)$. Indeed, the map $P(y)$ is continuous on $\left[t_{0}-h,+\infty\right)$ for each $y \in B C$, and for each $t \geqslant 2 t_{0}+h,\left(H_{2}\right)$ implies that

$$
\begin{aligned}
& |(P y)(t)| \\
& \leqslant\left|y\left(t_{0}\right)\right| e^{-\beta\left(t-t_{0}\right)} \\
& \quad+\frac{1}{\Gamma(\alpha)} \int_{t_{0}}^{t}(t-s)^{\alpha-1} e^{-\beta(t-s)}\left(K_{1}+K_{2}\left\|y_{s}\right\|\right) d s \\
& \leqslant\|\phi\| e^{-\beta\left(t-t_{0}\right)}+\frac{K_{1}+K_{2}\|y\|_{\infty}}{\Gamma(\alpha)} \\
& \quad \times\left(\int_{t_{0}}^{t-\left(t_{0}+h\right)}\left(t_{0}+h\right)^{\alpha-1} e^{-\beta(t-s)} d s\right. \\
& \left.\quad+\int_{t-\left(t_{0}+h\right)}^{t}(t-s)^{\alpha-1} d s\right) \\
& \leqslant\|\phi\|+\left(K_{1}+K_{2}\|y\|_{\infty}\right) \\
& \quad \times\left(\frac{\left(t_{0}+h\right)^{\alpha-1} e^{-\beta\left(t_{0}+h\right)}}{\beta \Gamma(\alpha)}+\frac{\left(t_{0}+h\right)^{\alpha}}{\Gamma(\alpha+1)}\right),
\end{aligned}
$$

for each $t \in\left[t_{0}, 2 t_{0}+h\right]$, we have

$$
|(P y)(t)| \leqslant\|\phi\|+\frac{\left(K_{1}+K_{2}\|y\|_{\infty}\right)\left(t_{0}+h\right)^{\alpha}}{\Gamma(\alpha+1)},
$$

and for any $t \in\left[t_{0}-h, t_{0}\right]$,

$$
|(P y)(t)| \leqslant\|\phi\| .
$$

Thus,

$$
\begin{aligned}
& \|P(y)\|_{\infty} \\
& \leqslant\|\phi\|+\left(K_{1}+K_{2}\|y\|_{\infty}\right) \\
& \quad \times\left(\frac{\left(t_{0}+h\right)^{\alpha-1} e^{-\beta\left(t_{0}+h\right)}}{\beta \Gamma(\alpha)}+\frac{\left(t_{0}+h\right)^{\alpha}}{\Gamma(\alpha+1)}\right),
\end{aligned}
$$

and consequently $P(y) \in B C$.

Next, we show that the operator $P$ is continuous and completely continuous, and there exists an open set $U \subset B C$ with $y \neq \lambda P(y)$ for $\lambda \in(0,1)$ and $y \in \partial U$.

Step 1 ( $P$ is continuous). Let $\left\{y_{n}\right\}$ be a sequence such that $y_{n} \rightarrow$ $y$ in $B C$. Then, there exist $R>0$ and $N>0$ such that

$$
\left\|y_{n}\right\|_{\infty}+\|y\|_{\infty}<R, \quad \forall n \geqslant N
$$

Let $\varepsilon>0$ be given. Since $\left(H_{4}\right)$ holds, there is a real number $T>0$ such that

$$
\frac{2}{\Gamma(\alpha)} \int_{t_{0}}^{t}(t-s)^{\alpha-1} e^{-\beta(t-s)}\left(k_{1}(s)+k_{2}(s) R\right) d s<\varepsilon,
$$

for all $t \geqslant T$. Now we consider the following two cases.

Case 1. If $t \geqslant T$, then it follows from $\left(H_{3}\right)$ and (30)-(31) that for $n$ sufficiently large

$$
\begin{aligned}
& \left|P y_{n}(t)-P y(t)\right| \\
& \leqslant\left|y_{n}\left(t_{0}\right)-y\left(t_{0}\right)\right| e^{-\beta\left(t-t_{0}\right)} \\
& \quad+\frac{1}{\Gamma(\alpha)} \int_{t_{0}}^{t}(t-s)^{\alpha-1} e^{-\beta(t-s)}\left|f\left(s, y_{n s}\right)-f\left(s, y_{s}\right)\right| d s \\
& \leqslant\left|y_{n}\left(t_{0}\right)-y\left(t_{0}\right)\right| \\
& \quad+\frac{2}{\Gamma(\alpha)} \int_{t_{0}}^{t}(t-s)^{\alpha-1} e^{-\beta(t-s)} \\
& \quad \times\left(k_{1}(s)+k_{2}(s) R\right) d s<2 \varepsilon .
\end{aligned}
$$

Case 2. If $t_{0} \leqslant t \leqslant T$, since $f$ is a continuous function, one has

$$
\begin{aligned}
& \left|P y_{n}(t)-P y(t)\right| \\
& \leqslant\left|y_{n}\left(t_{0}\right)-y\left(t_{0}\right)\right| \\
& \quad+\frac{1}{\Gamma(\alpha)} \int_{t_{0}}^{t}(t-s)^{\alpha-1} e^{-\beta(t-s)}\left|f\left(s, y_{n s}\right)-f\left(s, y_{s}\right)\right| d s \\
& \leqslant\left|y_{n}\left(t_{0}\right)-y\left(t_{0}\right)\right| \\
& \quad+\frac{\left(T-t_{0}\right)^{\alpha}}{\Gamma(\alpha+1)} \sup _{s \in\left[t_{0}, T\right]}\left|f\left(s, y_{n s}\right)-f\left(s, y_{s}\right)\right| .
\end{aligned}
$$


Note that $y_{n} \rightarrow y$ in BC. Hence, (32) and (33) imply that

$$
\left\|P\left(y_{n}\right)-P(y)\right\|_{\infty} \longrightarrow 0 \text { as } n \longrightarrow \infty .
$$

Step 2 (P maps bounded sets into bounded sets in BC). Indeed, it is enough to show that for any $\eta>0$, there exists a positive constant $\ell$ such that for each $y \in B_{\eta}=\left\{y \in B C:\|y\|_{\infty} \leqslant \eta\right\}$ one has $\|P(y)\|_{\infty} \leqslant \ell$. Let $y \in B_{\eta}$. Then, we have for each $t \geqslant 2 t_{0}+h$,

$$
\begin{aligned}
|(P y)(t)| \leqslant & \left|y\left(t_{0}\right)\right| e^{-\beta\left(t-t_{0}\right)} \\
& +\frac{1}{\Gamma(\alpha)} \int_{t_{0}}^{t}(t-s)^{\alpha-1} e^{-\beta(t-s)}\left|f\left(s, y_{s}\right)\right| d s \\
\leqslant & \eta+\frac{K_{1}+K_{2}\|y\|_{\infty}}{\Gamma(\alpha)} \int_{t_{0}}^{t}(t-s)^{\alpha-1} e^{-\beta(t-s)} d s \\
\leqslant & \eta+\left(K_{1}+K_{2} \eta\right) \\
& \times\left(\frac{\left(t_{0}+h\right)^{\alpha-1} e^{-\beta\left(t_{0}+h\right)}}{\beta \Gamma(\alpha)}+\frac{\left(t_{0}+h\right)^{\alpha}}{\Gamma(\alpha+1)}\right) \\
= & : \ell,
\end{aligned}
$$

and for each $t$ with $t_{0} \leqslant t \leqslant 2 t_{0}+h$,

$$
|(P y)(t)| \leqslant \eta+\left(K_{1}+K_{2} \eta\right) \frac{\left(t_{0}+h\right)^{\alpha}}{\Gamma(\alpha+1)}
$$

Hence, $\|P(y)\|_{\infty} \leqslant \ell$.

Step 3 ( $P$ maps bounded sets into equicontinuous sets on every compact subset $\left[t_{0}-h, b\right]$ of $\left.\left[t_{0}-h, \infty\right)\right)$. Let $t_{1}, t_{2} \in\left[t_{0}, b\right]$, $t_{1}<t_{2}$, and let $B_{\eta}$ be a bounded set of $B C$ as in Step 2. Let $y \in B_{\eta}$. Then, we have

$$
\begin{aligned}
& \left|(P y)\left(t_{2}\right)-(P y)\left(t_{1}\right)\right| \\
& \leqslant\left|y\left(t_{0}\right) e^{-\beta\left(t_{2}-t_{0}\right)}-y\left(t_{0}\right) e^{-\beta\left(t_{1}-t_{0}\right)}\right| \\
& \quad+\frac{1}{\Gamma(\alpha)} \int_{t_{0}}^{t_{1}} \mid\left(\left(t_{2}-s\right)^{\alpha-1} e^{-\beta\left(t_{2}-s\right)}\right. \\
& \left.\quad-\left(t_{1}-s\right)^{\alpha-1} e^{-\beta\left(t_{1}-s\right)}\right) f\left(s, y_{s}\right) \mid d s \\
& \quad+\frac{1}{\Gamma(\alpha)} \int_{t_{1}}^{t_{2}}\left|\left(t_{2}-s\right)^{\alpha-1} e^{-\beta\left(t_{2}-s\right)} f\left(s, y_{s}\right)\right| d s \\
& \leqslant\left|y\left(t_{0}\right)\right| e^{\beta t_{0}}\left|e^{-\beta t_{2}}-e^{-\beta t_{1}}\right|+\frac{K_{1}+K_{2} \eta}{\Gamma(\alpha+1)}\left(t_{2}-t_{1}\right)^{\alpha}
\end{aligned}
$$

$$
\begin{gathered}
+\frac{K_{1}+K_{2} \eta}{\Gamma(\alpha)} \int_{t_{0}}^{t_{1}}\left(\left(t_{1}-s\right)^{\alpha-1} e^{-\beta\left(t_{1}-s\right)}\right. \\
\left.-\left(t_{2}-s\right)^{\alpha-1} e^{-\beta\left(t_{2}-s\right)}\right) d s \\
\leqslant\left|y\left(t_{0}\right)\right| e^{\beta t_{0}}\left|e^{-\beta t_{2}}-e^{-\beta t_{1}}\right|+\frac{K_{1}+K_{2} \eta}{\Gamma(\alpha+1)}\left(t_{2}-t_{1}\right)^{\alpha} \\
+\frac{K_{1}+K_{2} \eta}{\Gamma(\alpha)} \int_{t_{0}}^{t_{1}}\left(\left(t_{1}-s\right)^{\alpha-1} e^{-\beta\left(t_{1}-s\right)}-\left(t_{2}-s\right)^{\alpha-1} e^{-\beta\left(t_{1}-s\right)}\right) d s \\
+\frac{K_{1}+K_{2} \eta}{\Gamma(\alpha)} \int_{t_{0}}^{t_{1}}\left(\left(t_{2}-s\right)^{\alpha-1} e^{-\beta\left(t_{1}-s\right)}\right. \\
\left.-\left(t_{2}-s\right)^{\alpha-1} e^{-\beta\left(t_{2}-s\right)}\right) d s .
\end{gathered}
$$

Observing that

$$
\begin{aligned}
& \frac{K_{1}+K_{2} \eta}{\Gamma(\alpha)} \\
& \quad \quad \times \int_{t_{0}}^{t_{1}}\left(\left(t_{1}-s\right)^{\alpha-1} e^{-\beta\left(t_{1}-s\right)}-\left(t_{2}-s\right)^{\alpha-1} e^{-\beta\left(t_{1}-s\right)}\right) d s \\
& \leqslant \frac{K_{1}+K_{2} \eta}{\Gamma(\alpha)} \int_{t_{0}}^{t_{1}}\left(\left(t_{1}-s\right)^{\alpha-1}-\left(t_{2}-s\right)^{\alpha-1}\right) d s \\
& \leqslant \frac{K_{1}+K_{2} \eta}{\Gamma(\alpha+1)}\left(\left(t_{1}-t_{0}\right)^{\alpha}-\left(t_{2}-t_{0}\right)^{\alpha}+\left(t_{2}-t_{1}\right)^{\alpha}\right) \\
& \leqslant \frac{K_{1}+K_{2} \eta}{\Gamma(\alpha+1)}\left(t_{2}-t_{1}\right)^{\alpha},
\end{aligned}
$$

from Taylor's theorem, we obtain

$$
\begin{aligned}
& \frac{K_{1}+K_{2} \eta}{\Gamma(\alpha)} \\
& \quad \times \int_{t_{0}}^{t_{1}}\left(\left(t_{2}-s\right)^{\alpha-1} e^{-\beta\left(t_{1}-s\right)}-\left(t_{2}-s\right)^{\alpha-1} e^{-\beta\left(t_{2}-s\right)}\right) d s \\
& \leqslant \frac{K_{1}+K_{2} \eta}{\Gamma(\alpha)}\left(t_{2}-t_{1}\right)^{\alpha-1} \int_{t_{0}}^{t_{1}}\left(e^{-\beta\left(t_{1}-s\right)}-e^{-\beta\left(t_{2}-s\right)}\right) d s \\
& \leqslant \frac{K_{1}+K_{2} \eta}{\beta \Gamma(\alpha)}\left(t_{2}-t_{1}\right)^{\alpha-1}\left(1-e^{-\beta\left(t_{2}-t_{1}\right)}\right) \\
& =\frac{K_{1}+K_{2} \eta}{\Gamma(\alpha)}\left(\left(t_{2}-t_{1}\right)^{\alpha}+\frac{o\left(t_{2}-t_{1}\right)}{t_{2}-t_{1}}\left(t_{2}-t_{1}\right)^{\alpha}\right),
\end{aligned}
$$

where $\lim _{t_{2}-t_{1} \rightarrow 0}\left(o\left(t_{2}-t_{1}\right) /\left(t_{2}-t_{1}\right)\right)=0$. By (37)-(39), we can conclude that

$$
\begin{aligned}
& \left|(P y)\left(t_{2}\right)-(P y)\left(t_{1}\right)\right| \\
& \leqslant \eta e^{\beta t_{0}}\left|e^{-\beta t_{2}}-e^{-\beta t_{1}}\right| \\
& \quad+\frac{2\left(K_{1}+K_{2} \eta\right)}{\Gamma(\alpha+1)}\left(t_{2}-t_{1}\right)^{\alpha} \\
& \quad+\frac{K_{1}+K_{2} \eta}{\Gamma(\alpha)}\left(\left(t_{2}-t_{1}\right)^{\alpha}+\frac{o\left(t_{2}-t_{1}\right)}{t_{2}-t_{1}}\left(t_{2}-t_{1}\right)^{\alpha}\right) .
\end{aligned}
$$


As $t_{1} \rightarrow t_{2}$, the right-hand side of the above inequality tends to zero. The equicontinuity for the cases $t_{1}<t_{2} \leqslant t_{0}$ and $t_{1} \leqslant t_{0} \leqslant t_{2}$ is obvious.

Step 4 ( $P$ maps bounded sets into equiconvergent sets). Let $y \in$ $B_{\eta}$. Then

$$
\begin{aligned}
& |(P y)(t)| \\
& \leqslant\left|y\left(t_{0}\right)\right| e^{-\beta\left(t-t_{0}\right)} \\
& \quad+\frac{1}{\Gamma(\alpha)} \int_{t_{0}}^{t}(t-s)^{\alpha-1} e^{-\beta(t-s)}\left|f\left(s, y_{s}\right)\right| d s \\
& \leqslant \\
& \quad \eta e^{-\beta\left(t-t_{0}\right)} \\
& \quad+\frac{1}{\Gamma(\alpha)} \int_{t_{0}}^{t}(t-s)^{\alpha-1} e^{-\beta(t-s)}\left(k_{1}(s)+k_{2}(s) \eta\right) d s .
\end{aligned}
$$

Therefore, $\left(H_{4}\right)$ implies that $|(P y)(t)|$ uniformly (with respect to $y \in B(\eta))$ converges to 0 as $t \rightarrow \infty$. As a consequence of Steps $1-4$, we can conclude that $P: B C \rightarrow B C$ is continuous and completely continuous.

Step 5 (a priori bounds). We now show that there exists an open set $U \subseteq B C$ with $y \neq \lambda P(y)$ for $\lambda \in(0,1)$ and $y \in \partial U$.

Let $y \in B C$ and $y=\lambda P(y)$ for some $0<\lambda<1$. Then, for each $t \in\left[t_{0}, \infty\right)$, we obtain

$$
\begin{aligned}
y(t)=\lambda\left[y\left(t_{0}\right) e^{-\beta\left(t-t_{0}\right)}\right. & \\
& \left.+\frac{1}{\Gamma(\alpha)} \int_{t_{0}}^{t}(t-s)^{\alpha-1} e^{-\beta(t-s)} f\left(s, y_{s}\right) d s\right] .
\end{aligned}
$$

By $\left(H_{3}\right)$, we have that for all $\theta \in[-h, 0]$ and $t \geqslant t_{0}+h$,

$$
\begin{aligned}
& |y(t+\theta)| \\
& \leqslant\left|y\left(t_{0}\right)\right| e^{-\beta\left(t+\theta-t_{0}\right)} \\
& \quad+\frac{1}{\Gamma(\alpha)} \int_{t_{0}}^{t+\theta}(t+\theta-s)^{\alpha-1} e^{-\beta(t+\theta-s)}\left|f\left(s, y_{s}\right)\right| d s \\
& \leqslant\left|y\left(t_{0}\right)\right| e^{-\beta\left(t+\theta-t_{0}\right)} \\
& \quad+\frac{1}{\Gamma(\alpha)} \int_{t_{0}}^{t+\theta}(t+\theta-s)^{\alpha-1} e^{-\beta(t+\theta-s)}\left(K_{1}+K_{2}\left\|y_{s}\right\|\right) d s,
\end{aligned}
$$

and thus

$\left\|y_{t}\right\|$

$$
\begin{aligned}
\leqslant & \left|y\left(t_{0}\right)\right| e^{-\beta\left(t-h-t_{0}\right)} \\
& +\frac{1}{\Gamma(\alpha)} \int_{t_{0}}^{t}(t-s)^{\alpha-1} e^{-\beta(t-h-s)}\left(K_{1}+K_{2}\left\|y_{s}\right\|\right) d s .
\end{aligned}
$$

From the arguments in (26)-(27), we can conclude that for each $t \in\left[t_{0}, \infty\right)$,

$$
\begin{aligned}
& \frac{1}{\Gamma(\alpha)} \int_{t_{0}}^{t}(t-s)^{\alpha-1} e^{-\beta(t-s)} d s \\
& \quad \leqslant \frac{\left(t_{0}+h\right)^{\alpha-1} e^{-\beta\left(t_{0}+h\right)}}{\beta \Gamma(\alpha)}+\frac{\left(t_{0}+h\right)^{\alpha}}{\Gamma(\alpha+1)}=: R_{1} .
\end{aligned}
$$

Hence,

$$
\begin{aligned}
e^{\beta t}\left\|y_{t}\right\| & \\
\leqslant & \|\phi\| e^{\beta\left(h+t_{0}\right)}+e^{\beta(h+t)} K_{1} R_{1} \\
& +\frac{K_{2} e^{\beta h}}{\Gamma(\alpha)} \int_{t_{0}}^{t}(t-s)^{\alpha-1} e^{\beta s}\left\|y_{s}\right\| d s .
\end{aligned}
$$

Let $R_{2}=\|\phi\| e^{\beta\left(h+t_{0}\right)}+e^{\beta h} K_{1} R_{1}$. Then, from Lemma 2, there exists $K$ such that we have for all $t \geqslant t_{0}+h$,

$$
\left\|y_{t}\right\| \leqslant R_{2}+\frac{K K_{2} R_{2} e^{\beta h}}{\Gamma(\alpha+1)}\left(t-t_{0}\right)^{\alpha} e^{-\beta t} .
$$

Since $\lim _{t \rightarrow \infty}\left(t-t_{0}\right)^{\alpha} e^{-\beta t}=0$, there exists $R_{3}>0$ such that

$$
\|y\|_{\infty} \leqslant R_{3} \text {. }
$$

Set

$$
U=\left\{y \in B C:\|y\|_{\infty}<R_{3}+1\right\} .
$$

$P: U \rightarrow B C$ is continuous and completely continuous. From the choice of $U$, there is no $y \in \partial U$ such that $y=\lambda P(y)$, for $\lambda \in(0,1)$. As a consequence of Leray-Schauder fixed-point theorem, we deduce that $P$ has a fixed point $y$ in $U$.

Step 6 (uniform asymptotic stability of solutions). Let $B \subset$ $C([-h, 0], \mathbb{R})$ be bounded; that is, there exists $d \geqslant 0$ such that

$$
\|\psi\|=\sup _{\theta \in[-h, 0]}|\psi(\theta)| \leqslant d \quad \forall \psi \in B .
$$

From the similar arguments in Step 4, we can deduce that there exists $R_{4}>0$ such that for all solutions $y\left(t, t_{0}, \phi\right)$ of IVP (1) with initial data $\phi \in B$, we have

$$
\|y\|_{\infty} \leqslant R_{4}, \quad \forall \phi \in B .
$$

Now we consider two solutions $x=x(t)$ and $y=y(t)$ of IVP (1) corresponding to the initial values $\psi$ and $\phi$. Note that for all $t \geqslant t_{0}$,

$$
\begin{aligned}
& |x(t)-y(t)| \\
& \leqslant\left|x\left(t_{0}\right)-y\left(t_{0}\right)\right| e^{-\beta\left(t-t_{0}\right)} \\
& \quad+\frac{1}{\Gamma(\alpha)} \int_{t_{0}}^{t}(t-s)^{\alpha-1} e^{-\beta(t-s)}\left(\left|f\left(s, x_{s}\right)\right|+\left|f\left(s, y_{s}\right)\right|\right) d s \\
& \leqslant 2 d e^{-\beta\left(t-t_{0}\right)} \\
& \quad+\frac{2}{\Gamma(\alpha)} \int_{t_{0}}^{t}(t-s)^{\alpha-1} e^{-\beta(t-s)}\left(k_{1}(s)+k_{2}(s) R_{4}\right) d s .
\end{aligned}
$$


Then, the proof of uniform asymptotic stability of solutions can be done by making use of $\left(\mathrm{H}_{4}\right)$ and (52).

The proof of Theorem 5 is completed.

\section{Examples}

Example 1. Consider the fractional functional differential equation

$$
\begin{gathered}
D^{1 / 2}\left[y(t) e^{t}\right]=\frac{e^{2 t}}{8\left(e^{t}+e^{-t}\right)} \sin ^{4}(y(t-1))+e^{t}, \quad t \geqslant 0 \\
y(t)=\phi(t), \quad-1 \leqslant t \leqslant 0
\end{gathered}
$$

where $f\left(t, y_{t}\right)=\left(e^{t} / 8\left(e^{t}+e^{-t}\right)\right) \sin ^{4}(y(t-1))+1$. It is clear that condition $\left(H_{0}\right)$ holds. Let $x_{t}, y_{t} \in C([-1,0], \mathbb{R})$. Then for all $t \in[0, \infty)$, we have

$$
\begin{aligned}
& \left|f\left(t, x_{t}\right)-f\left(t, y_{t}\right)\right| \\
& \quad=\frac{e^{t}}{8\left(e^{t}+e^{-t}\right)}\left|\sin ^{4}(x(t-1))-\sin ^{4}(y(t-1))\right| \\
& \quad \leqslant \frac{e^{t}}{2\left(e^{t}+e^{-t}\right)}|x(t-1)-y(t-1)| \\
& \quad \leqslant \frac{1}{2}|x(t-1)-y(t-1)| .
\end{aligned}
$$

On the other hand, note that $f(t, 0)=1$ for each $t \in[0, \infty)$ and $(1 / 2)\left(e^{-1} / \Gamma(1 / 2)+1 / \Gamma(3 / 2)\right)<1$. Hence, conditions $\left(H_{1}\right)$ and (10) hold. By Theorem 4, we conclude that IVP (53) has a unique solution in the space $B C([-1, \infty), \mathbb{R})$, and the solution of IVP (53) is uniformly asymptotically stable.

Example 2. Consider the fractional functional differential equation

$$
\begin{gathered}
D^{1 / 2}\left[y(t) e^{t}\right]=10 e^{t}(t+1)^{-3 / 4} \frac{y(t-1)}{1+|y(t-1)|}, \quad t \geqslant 0, \\
y(t)=\phi(t), \quad-1 \leqslant t \leqslant 0,
\end{gathered}
$$

where $f\left(t, y_{t}\right)=10(t+1)^{-3 / 4}(y(t-1) /(1+|y(t-1)|))$. It is easy to see that condition $\left(H_{2}\right)$ holds. Let $y_{t} \in C([-1,0], \mathbb{R})$. Then, for all $t \in[0, \infty)$, we find that

$$
\begin{aligned}
& \left|f\left(t, y_{t}\right)\right| \\
& \quad=\left|10(t+1)^{-3 / 4} \frac{y(t-1)}{1+|y(t-1)|}\right| \leqslant 10(t+1)^{-3 / 4}|y(t-1)|,
\end{aligned}
$$

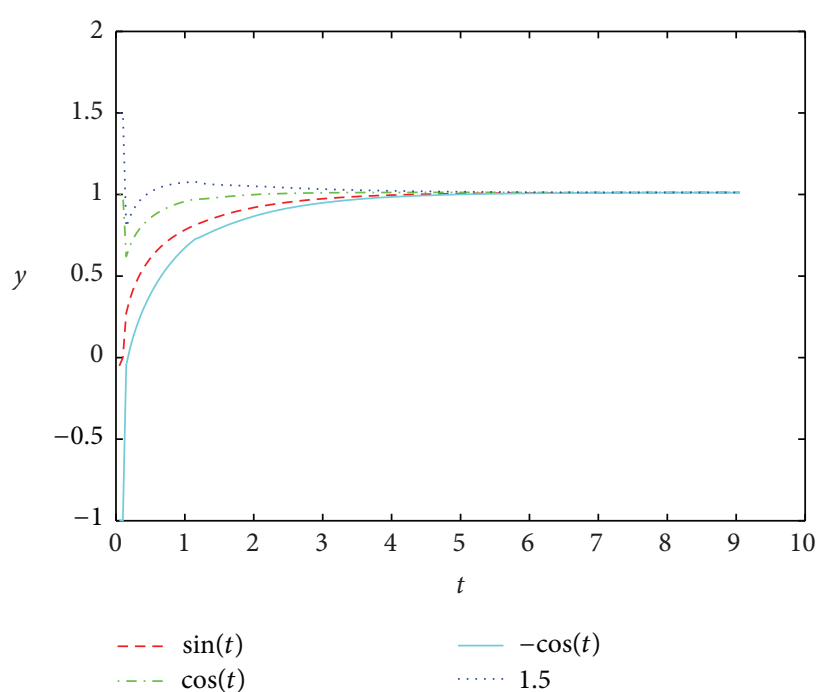

FIgURE 1: The numerical solutions of Example 1 with the initial conditions $\phi(t)=\sin (t), \cos (t),-\cos (t)$, and 1.5 , respectively.

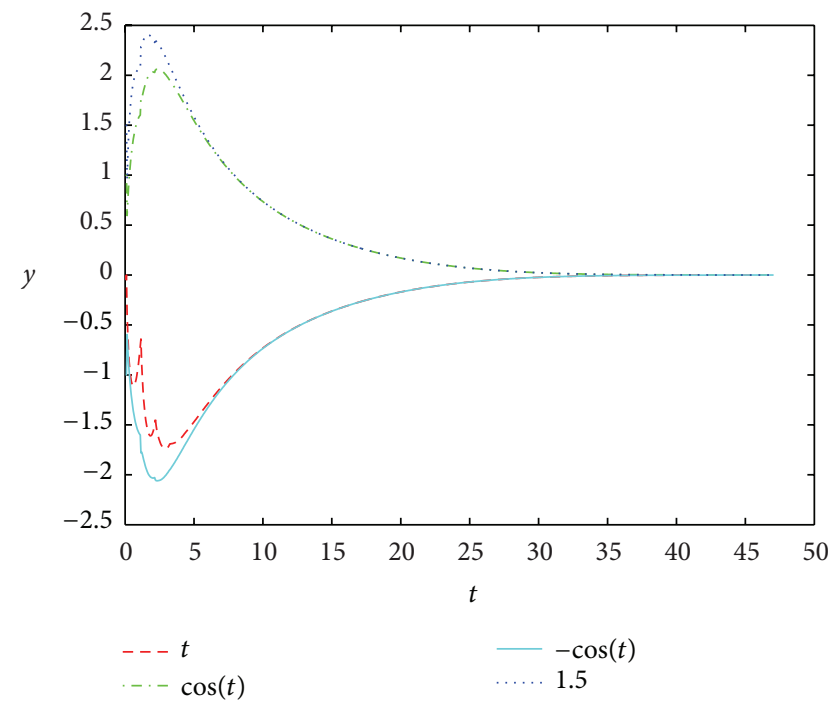

FIgURE 2: The numerical solutions of Example 2 with the initial conditions $\phi(t)=t, \cos (t),-\cos (t)$, and 1.5 , respectively.

where $10(t+1)^{-3 / 4} \in B C\left([0, \infty), \mathbb{R}_{+}\right)$with $\sup _{t \geqslant 0} 10(t+$ $1)^{-3 / 4}=10$ and

$$
\begin{aligned}
& \frac{1}{\Gamma(1 / 2)} \int_{0}^{t}(t-s)^{-1 / 2} e^{-(t-s)} 10(s+1)^{-3 / 4} d s \\
& \quad \leqslant \frac{10}{\Gamma(1 / 2)} \int_{0}^{t}(t-s)^{-1 / 2} s^{-3 / 4} d s \\
& \quad=\frac{10 \Gamma(1 / 4)}{\Gamma(3 / 4)} t^{-1 / 4} \longrightarrow 0 \text { as } t \rightarrow \infty .
\end{aligned}
$$

Thus, conditions $\left(H_{3}\right)$ and $\left(H_{4}\right)$ hold, and the global existence and the uniform asymptotic stability of solutions of IVP (55) can be obtained by applying Theorem 5 . 
By using the algorithm given in [19], we numerically simulate Example 1 with the initial conditions $\phi(t)=\sin (t)$, $\cos (t),-\cos (t), 1.5$, and Example 2 with $\phi(t)=t, \cos (t)$, $-\cos (t), 1.5$; see Figures 1 and 2 . From the numerical results, it can be noted that both of the solutions of Examples 1 and 2 converge uniformly, and the solutions of Example 1 converge faster than the ones of Example 2. The numerical results confirm the theoretical analysis.

\section{Conflict of Interests}

The authors declare that there is no conflict of interests regarding the publication of this paper.

\section{Acknowledgments}

This research is supported by the National Natural Science Foundation of China under Grants nos. 10801066 and 11271173 and the Fundamental Research Funds for the Central Universities under Grant nos. lzujbky-2011-47 and lzujbky2012-k26. The project is sponsored by the Scientific Research Foundation for the Returned Overseas Chinese Scholars, State Education Ministry.

\section{References}

[1] T. Caraballo, P. Marín-Rubio, and J. Valero, "Autonomous and non-autonomous attractors for differential equations with delays," Journal of Differential Equations, vol. 208, no. 1, pp. 9-41, 2005.

[2] J. K. Hale and S. M. Verduyn Lunel, Introduction to Functional Differential Equations, Springer, New York, NY, USA, 1993.

[3] Y. Kuang, Delay Differential Equations with Applications in Population Dynamics, vol. 191 of Mathematics in Science and Engineering, Academic Press, New York, NY, USA, 1993.

[4] M. C. Mackey and L. Glass, "Oscillation and chaos in physiological control systems," Science, vol. 197, pp. 287-289, 1977.

[5] M. Benchohra, J. Henderson, S. K. Ntouyas, and A. Ouahab, "Existence results for fractional order functional differential equations with infinite delay," Journal of Mathematical Analysis and Applications, vol. 338, no. 2, pp. 1340-1350, 2008.

[6] M. Benchohra and B. A. Slimani, "Partial neutral functional hyperbolic differential equations with Caputo fractional derivative," Nonlinear Analysis Forum, vol. 15, pp. 143-151, 2010.

[7] K. Diethelm and N. J. Ford, "Analysis of fractional differential equations," Journal of Mathematical Analysis and Applications, vol. 265, no. 2, pp. 229-248, 2002.

[8] K. Diethelm, The Analysis of Fractional Differential Equations. An Application-Oriented Exposition Using Differential Operators of Caputo Type, vol. 2004 of Lecture Notes in Mathematics, Springer, Berlin, Germany, 2010.

[9] A. A. Kilbas and J. J. Trujillo, "Differential equations of fractional order: methods, results and problems. II," Applicable Analysis, vol. 81, no. 2, pp. 435-493, 2002.

[10] S. A. Messaoudi, B. Said-Houari, and N. Tatar, "Global existence and asymptotic behavior for a fractional differential equation," Applied Mathematics and Computation, vol. 188, no. 2, pp. 19551962, 2007.
[11] I. Podlubny, Fractional Differential Equations, vol. 198 of Mathematics in Science and Engineering, Academic Press, San Diego, Calif, USA, 1999.

[12] F. Chen and Y. Zhou, "Attractivity of fractional functional differential equations," Computers \& Mathematics with Applications, vol. 62, no. 3, pp. 1359-1369, 2011.

[13] F. Chen, J. J. Nieto, and Y. Zhou, "Global attractivity for nonlinear fractional differential equations," Nonlinear Analysis. Real World Applications, vol. 13, no. 1, pp. 287-298, 2012.

[14] B. C. Dhage, "Local asymptotic attractivity for nonlinear quadratic functional integral equations," Nonlinear Analysis. Theory, Methods \& Applications, vol. 70, no. 5, pp. 1912-1922, 2009.

[15] B. C. Dhage, "Global attractivity results for nonlinear functional integral equations via a Krasnoselskii type fixed point theorem," Nonlinear Analysis. Theory, Methods \& Applications, vol. 70, no. 7, pp. 2485-2493, 2009.

[16] B. C. Dhage and V. Lakshmikantham, "On global existence and attractivity results for nonlinear functional integral equations," Nonlinear Analysis. Theory, Methods \& Applications, vol. 72, no. 5, pp. 2219-2227, 2010.

[17] J. Banaś and B. C. Dhage, "Global asymptotic stability of solutions of a functional integral equation," Nonlinear Analysis. Theory, Methods \& Applications, vol. 69, no. 7, pp. 1945-1952, 2008.

[18] D. Henry, Geometric Theory of Semilinear Parabolic Partial Differential Equations, Springer, Berlin, Germany, 1989.

[19] W. H. Deng, "Numerical algorithm for the time fractional Fokker-Planck equation," Journal of Computational Physics, vol. 227, no. 2, pp. 1510-1522, 2007. 


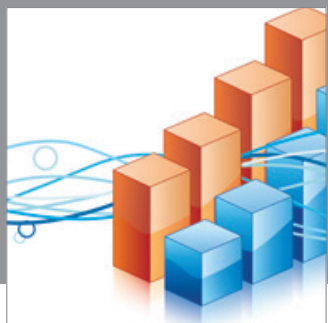

Advances in

Operations Research

mansans

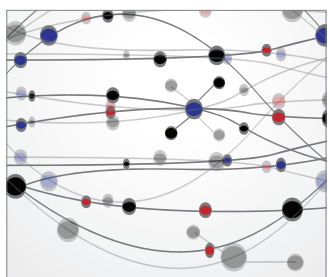

The Scientific World Journal
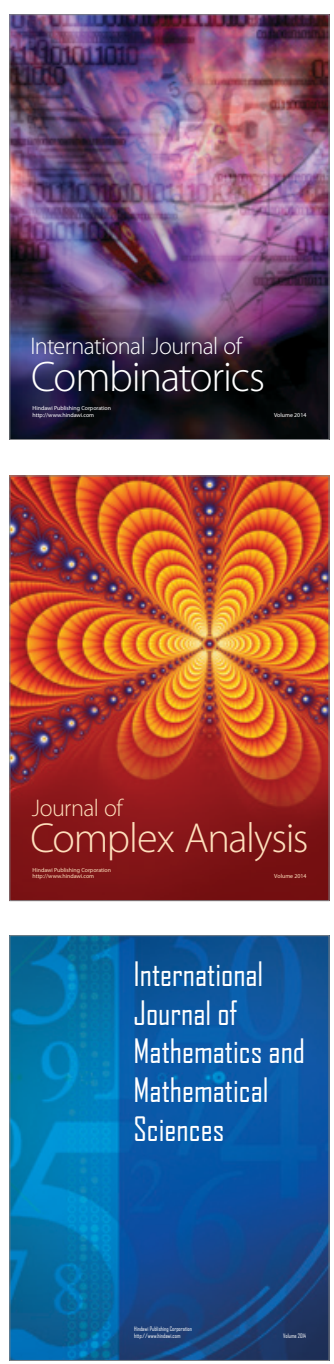
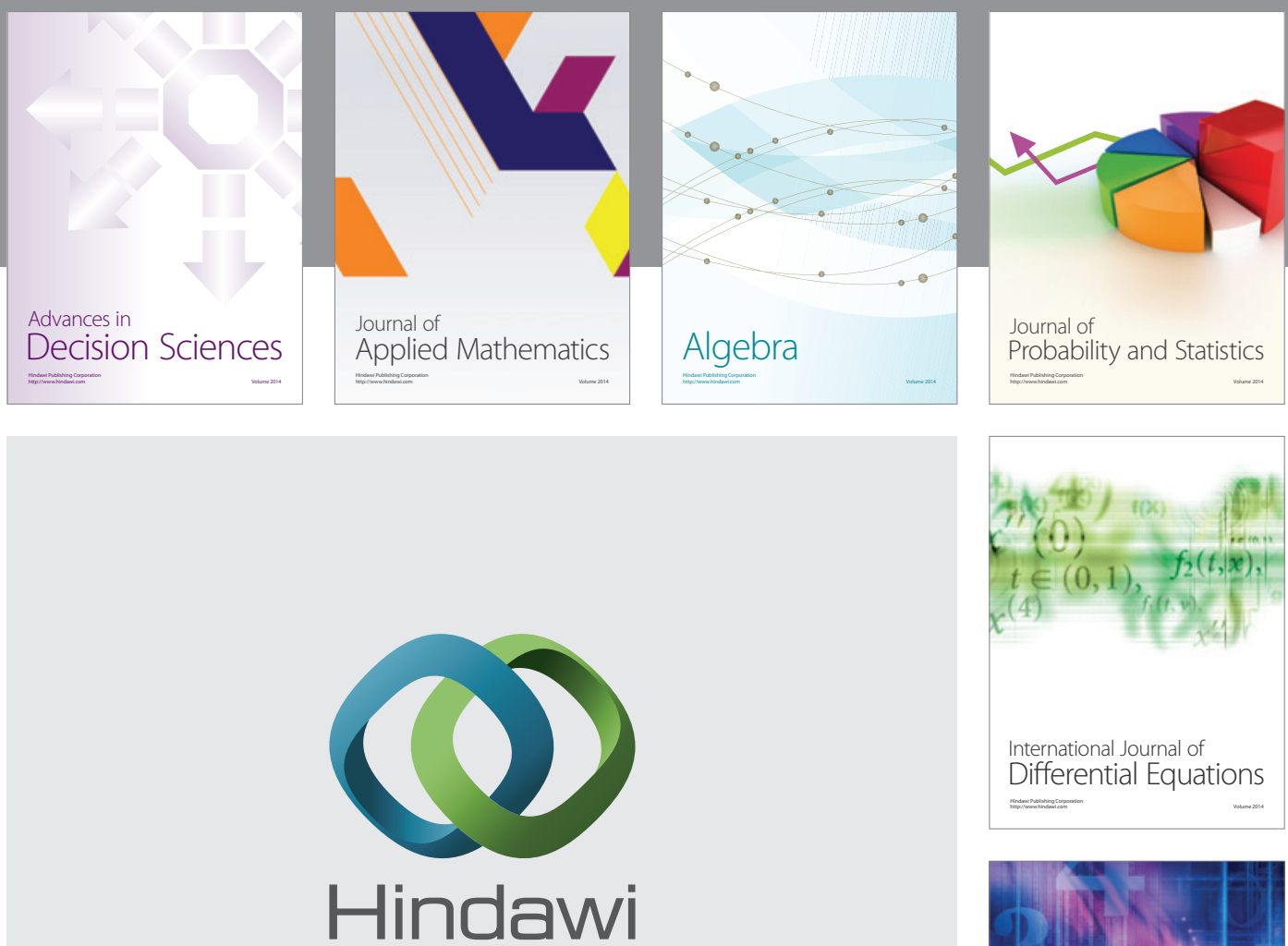

Submit your manuscripts at http://www.hindawi.com
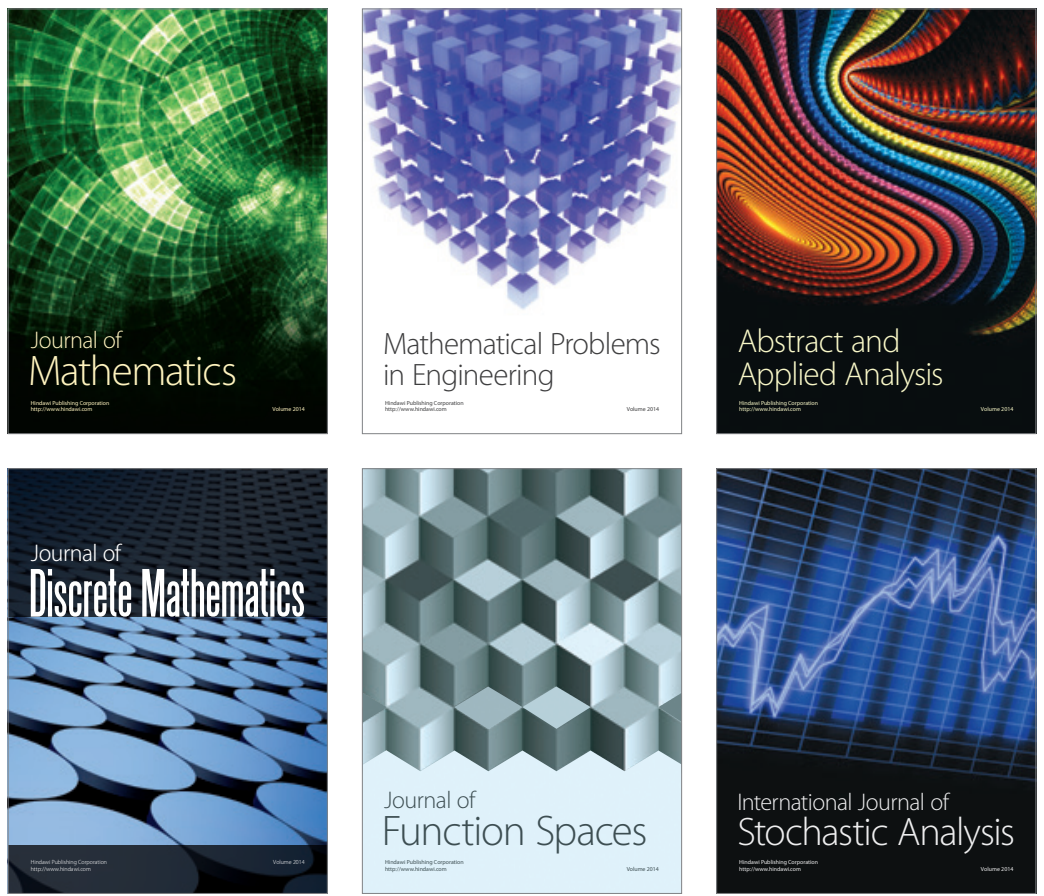

Journal of

Function Spaces

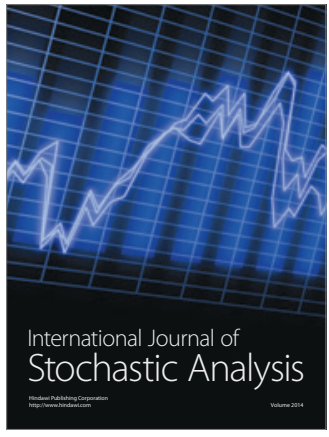

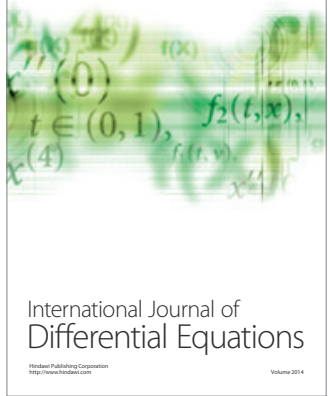
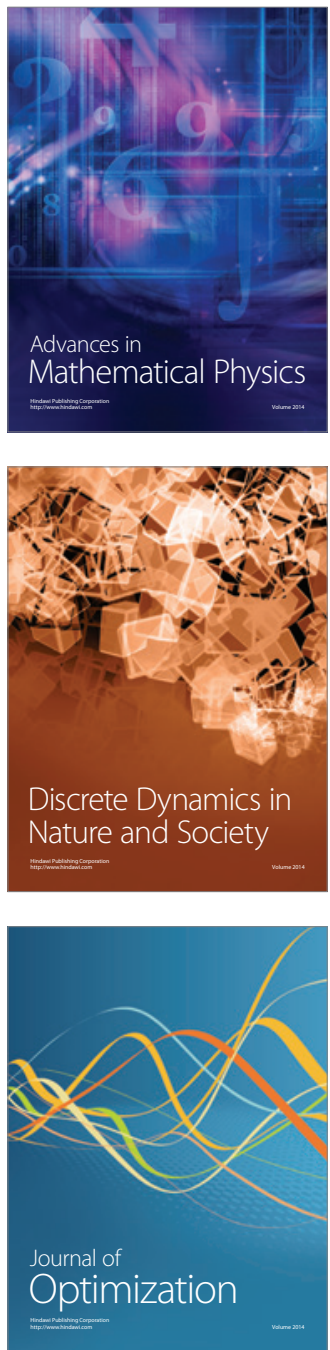